\title{
Bleeding associated with coadministration of rivaroxaban and clarithromycin
}

\author{
Michael Fralick MD, David N. Juurlink MD PhD, Theodore Marras MD MSc
}

A 65-year-old man presented to hospital with hemoptysis, epistaxis, headache and syncope. His medical history included antiphospholipid antibody syndrome with recurrent deep vein thrombosis, most recently 10 years earlier. He also had mixed restrictive-obstructive lung disease, myasthenia gravis, autoimmune hemolytic anemia and idiopathic thrombocytopenic purpura. The latter two disorders were quiescent when he was assessed 10 days before the current admission. His medications included pantoprazole $40 \mathrm{mg} / \mathrm{d}$, prednisone $5 \mathrm{mg} / \mathrm{d}$, rituximab $650 \mathrm{mg}$ intravenously every three months (last dose three months previously), cotrimoxazole one double-strength tablet every other day (for Pneumocystis jiroveci prophylaxis) and tiotropium $18 \mu \mathrm{g} / \mathrm{d}$. To avoid the inconvenience of weekly monitoring of international normalized ratio, rivaroxaban $20 \mathrm{mg} / \mathrm{d}$ had been started four months before the current admission, following 12 years of uneventful warfarin therapy. Three days before presenting to hospital, the patient self-initiated clarithromycin $500 \mathrm{mg}$ twice daily for progressive sore throat, cough and dyspnea. Clarithromycin was one of two antibiotics that the patient's respirologist had maintained on file for years with the patient's pharmacy for rapid treatment of pulmonary exacerbations, with the most recent prescription renewal occurring before initiation of rivaroxaban. The patient had successfully used this antibiotic for exacerbations in the past, but the hematologist who prescribed rivaroxaban may not have been aware of this occasional use of clarithromycin.

On physical examination, the patient's vital signs were stable. The results of a cardiovascular examination were normal, and a respiratory examination showed mild respiratory distress and bilateral wheeze. The patient was agitated and disoriented, but other findings from the neurologic examination were normal.

The results of routine laboratory studies were largely normal (see Appendix 1, available at www.cmaj.ca/lookup/suppl/doi:10.1503/cmaj. 150580/-/DC1). Prothrombin time was elevated (16.5 [normal 11-15.5] s). Results of testing for antineutrophil cytoplasmic and antiglomerular basement membrane antibodies were negative, and complement levels were normal. Culture of sputum and blood, acid-fast bacilli staining, nasopharyngeal swab and urine testing for Legionella antigen all yielded negative results. Computed tomography (CT) of the thorax showed parenchymal opacities consistent with pulmonary hemorrhage (Figure 1). There were no pulmonary emboli. A CT head scan showed a $4-\mathrm{cm} \times 2.7-\mathrm{cm}$ intracranial hemorrhage in the left frontal lobe, with bilateral frontal subarachnoid hemorrhage (Figure 2).

Given the lengthy interval $(33 \mathrm{~h})$ between the last dose of rivaroxaban and presentation to hospital, the patient's physicians opted not to administer prothrombin complex concentrate. Measurement of anti-factor Xa level was ordered, but the result was not available until day 7 of the admission. This anti-factor Xa level, in a sample obtained 33 hours after the last dose of rivaroxaban, was elevated $(537 \mu \mathrm{g} / \mathrm{L})$; the normal trough level, typically measured 24 hours after drug administration, ranges from 8 to $150 \mu \mathrm{g} / \mathrm{L} .{ }^{1,2}$ Clarithromycin was stopped, and the patient received heparin 5000 units twice daily for venous thromboembolism prophylaxis while he was in hospital. Blood

\section{KEY POINTS}

- Rivaroxaban is metabolized by the cytochrome P450 3A4 isozyme (CYP3A4) and is a substrate for P-glycoprotein.

- Strong inhibitors of CYP3A4 and P-glycoprotein (e.g., ritonavir, ketoconazole, itraconazole, posaconazole and clarithromycin) should be avoided in patients receiving rivaroxaban.

- Patients with severe renal failure (creatinine clearance $<30 \mathrm{~mL} / \mathrm{min}$ ) or severe hepatic failure should avoid rivaroxaban when clinically appropriate.

- Rivaroxaban-related bleeding is managed supportively, and prothrombin complex concentrate should be considered for life-threatening bleeding. 
transfusion was not required, the patient had no persistent neurologic deficits, and his agitation resolved. The intracranial hemorrhage was treated conservatively, and repeat CT head scan on day 7 showed no worsening of the hemorrhage. On day 14 of the admission, rivaroxaban was restarted by the hematologist.

\section{Discussion}

The direct factor $\mathrm{Xa}$ inhibitor rivaroxaban is an oral anticoagulant approved for the prevention and treatment of venous thromboembolism and for stroke prophylaxis in patients with nonvalvu-

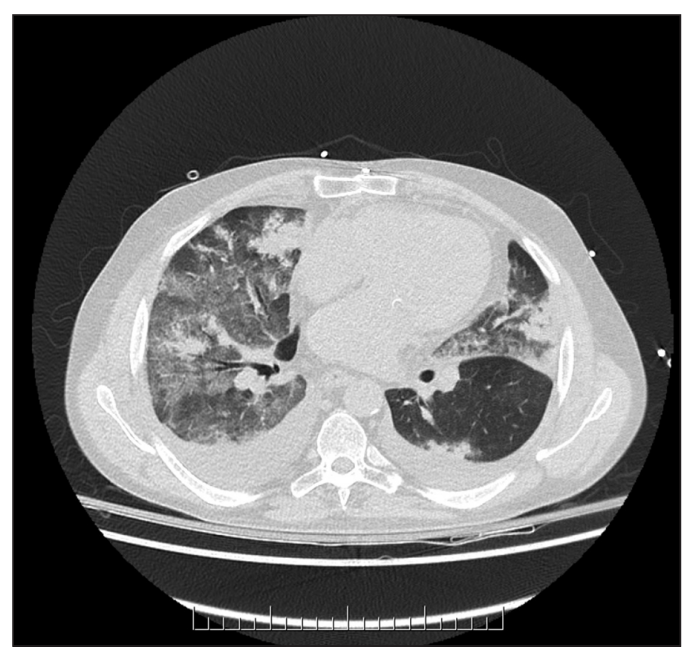

Figure 1: Computed tomography of the thorax of a 65-year-old man with hemoptysis, showing bilateral parenchymal opacities, including ground-glass opacity and consolidation, consistent with pulmonary hemorrhage. Bilateral pleural effusions are also present.

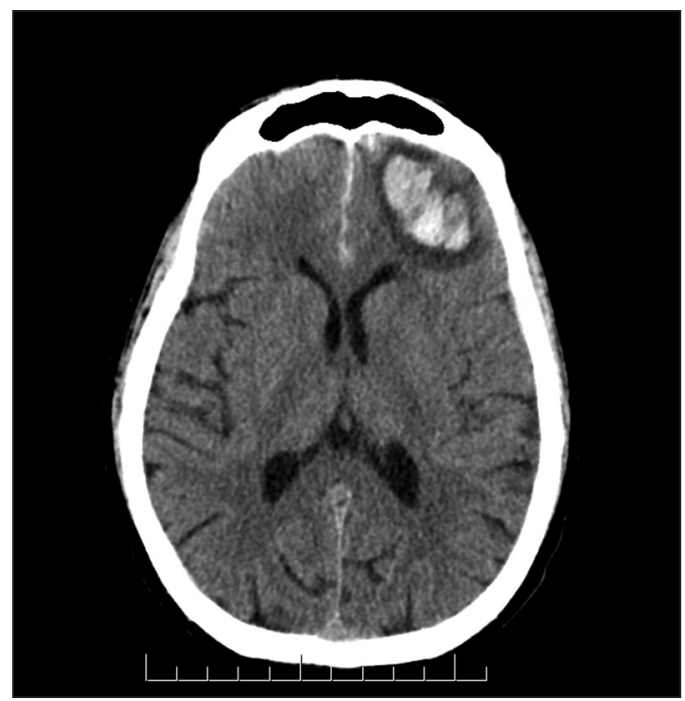

Figure 2: Computed tomography of the head, showing intracranial hemorrhage in the left frontal lobe. lar atrial fibrillation. ${ }^{1}$ The most common adverse effect is hemorrhage, with up to $5 \%$ of patients experiencing major bleeding. ${ }^{3}$

One advantage of rivaroxaban is that routine coagulation monitoring is not required. Prothrombin time and activated prothrombin time do not correlate well with plasma levels of this drug. ${ }^{1}$ Although prolonged prothrombin time may indicate the presence of rivaroxaban, the degree of prolongation does not predict the amount of drug present. ${ }^{1}$ Anti-factor Xa levels correlate well with plasma concentrations of rivaroxaban, but the availability of this test is limited. ${ }^{1,2}$

We have described a potential interaction between clarithromycin and rivaroxaban. At 33 hours after this patient's last dose of rivaroxaban, his anti-factor Xa level was $537 \mu \mathrm{g} / \mathrm{L}$, consistent with a marked increase in exposure, despite preservation of renal and hepatic function and a low baseline risk of bleeding. ${ }^{4}$

Rivaroxaban has moderate oral bioavailability (66\% for a $20-\mathrm{mg}$ dose), is absorbed rapidly and has an elimination half-life of about 10 hours. ${ }^{1}$ It is metabolized primarily by cytochrome $\mathrm{P} 450$ isoenzyme 3A4 (CYP3A4) to inactive metabolites and is a substrate for both CYP3A4 and P-glycoprotein. CYP3A4, one of the most common P450 isozymes, is found primarily in the liver and intestine.

Elimination of rivaroxaban occurs through hepatobiliary and renal routes. ${ }^{1}$ Hepatobiliary elimination occurs via the multidrug efflux transporter P-glycoprotein, which binds rivaroxaban and facilitates its gastrointestinal excretion. P-glycoprotein is also expressed in the small intestine, where it opposes absorption of rivaroxaban. ${ }^{5}$ Rivaroxaban is also eliminated unchanged in the urine via glomerular filtration and active secretion, mediated by P-glycoprotein. ${ }^{1,5,6}$

\section{Interactions with rivaroxaban}

Rivaroxaban is a substrate for CYP3A4 and Pglycoprotein, both of which are subject to inhibition or induction by many drugs. Clarithromycin is a strong inhibitor of both. ${ }^{5,7}$ Because CYP3A4 plays a major role in the oxidative metabolism of rivaroxaban, inhibition of this isozyme can result in increased plasma concentrations of the drug. ${ }^{7}$ Inhibition of P-glycoprotein in the liver limits transport of rivaroxaban into bile, while inhibition in the intestine and proximal tubule results in increased absorption and decreased elimination of rivaroxaban, respectively. ${ }^{1,7}$

In one study of healthy volunteers, clarithromycin increased the mean area under the curve and maximum concentration of rivaroxaban by up to $64 \%$ and $52 \%$, respectively. ${ }^{7}$ However, 
patients in that study received a 10-mg dose of rivaroxaban, and on only one day did they receive both medications together. Our patient received rivaroxaban $20 \mathrm{mg} / \mathrm{d}$, in addition to three days of clarithromycin therapy. Moreover, none of his other medications are strong inhibitors of CYP3A4 or P-glycoprotein, and none are independently associated with hemorrhage.

In summary, clarithromycin inhibits the metabolism of rivaroxaban, enhances systemic absorption of the drug and reduces its elimination. It is highly probable that the patient's hemoptysis, epistaxis and intracranial hemorrhage were the result of a drug interaction between rivaroxaban and clarithromycin. ${ }^{8}$

\section{Preventing drug interactions involving rivaroxaban}

Coadministration of rivaroxaban with strong inhibitors of both CYP3A4 and P-glycoprotein, including ritonavir, ketoconazole, itraconazole, posaconazole and clarithromycin, is not recommended. ${ }^{7}$ Pharmacokinetic studies have suggested that a drug that is a P-glycoprotein inhibitor or a moderate CYP3A4 inhibitor (or both) can be prescribed with rivaroxaban, but this should be done with caution. ${ }^{7}$ Specifically, if alternative options are available, then they should be prescribed. When no alternative exists, the patient should be counselled about signs of bleeding, and the dose and duration of the interacting medication should be limited. Clinicians may also consider prescrib- ing a lower dose of rivaroxaban, although this recommendation is based on pragmatic considerations and pharmacokinetic data rather than clinical studies. ${ }^{1,7}$ Commonly prescribed inhibitors of CYP3A4 and P-glycoprotein are listed in Box 1,7,9 along with suggestions for clinicians. More complete lists can be found elsewhere (e.g., University of Indiana Department of Medicine [http://medicine.iupui.edu/clinpharm/ddis/clinical -table]; Epocrates app, Athenahealth Inc.; Micromedex databases, Truven Health Analytics Inc.). For complex scenarios involving long-term coprescription of rivaroxaban with inhibitors of CYP3A4 or P-glycoprotein (or both), consultation with a pharmacist is advised.

Monitoring with anti-factor Xa assays is generally not recommended, for three reasons. First, the availability of the test is limited because of the detailed calibration required. ${ }^{1}$ Second, interpreting the results can be challenging unless the exact time of last drug administration is known. Third, a specific cut-off that correlates with clinically important bleeding has not yet been determined. ${ }^{1}$ Experts recommend ordering measurement of anti-factor Xa level or prothrombin time before urgent surgery or procedures and for lifethreatening bleeding, acute renal failure or suspected overdose. ${ }^{1}$ In these situations, elevated anti-factor Xa level or prothrombin time can help to confirm the presence of rivaroxaban and can potentially influence strategies to achieve hemostasis and timing of the subsequent dose.

Box 1: Clinical scenarios with the potential to increase bleeding risk in patients who are taking rivaroxaban*

\begin{tabular}{|c|c|c|}
\hline Scenario & Details & Suggested management $†$ \\
\hline $\begin{array}{l}\text { Combined P-gp and strong } \\
\text { CYP3A4 inhibitor }\end{array}$ & $\begin{array}{l}\text { Antifungals: posaconazole, itraconazole, ketoconazole } \\
\text { Macrolide: clarithromycin }\end{array}$ & Avoid these medications \\
\hline $\begin{array}{l}\text { Combined P-gp and/or } \\
\text { moderate CYP3A4 inhibitor }\end{array}$ & $\begin{array}{l}\text { Antimicrobials: erythromycin, ciprofloxacin, fluconazole, } \\
\text { voriconazole, isoniazid } \\
\text { Antidepressants: fluoxetine, fluvoxamine, sertraline } \\
\text { Antihypertensives: verapamil, diltiazem } \\
\text { Antiarrhythmic: amiodarone } \\
\text { Antivirals for HIV: atazanavir, efavirenz, telaprevir }\end{array}$ & $\begin{array}{l}\text { Use these medications with } \\
\text { caution; consider a lower dose of } \\
\text { rivaroxaban }\end{array}$ \\
\hline $\begin{array}{l}\text { Concomitant use of drugs } \\
\text { affecting hemostasis }\end{array}$ & $\begin{array}{l}\text { Anti-inflammatory agents: NSAIDs } \\
\text { Antiplatelet agents: ASA, clopidogrel, prasugrel, ticagrelor }\end{array}$ & $\begin{array}{l}\text { Use these medications with } \\
\text { caution; re-evaluate need for } \\
\text { antiplatelet therapy }\end{array}$ \\
\hline Dietary products & Grapefruit juice, dietary quinine & Use these products with caution \\
\hline \multirow[t]{2}{*}{ Renal failure } & $\mathrm{CrCl} 30-49 \mathrm{~mL} / \mathrm{min}$ & $\begin{array}{l}\text { Decrease the dose of rivaroxaban } \\
\text { (to } 15 \mathrm{mg} / \mathrm{d} \text { ) }\end{array}$ \\
\hline & $\mathrm{CrCl}<30 \mathrm{~mL} / \mathrm{min}$ & Avoid use of rivaroxaban \\
\hline Liver failure & Child-Pugh class B and C & Avoid use of rivaroxaban \\
\hline \multicolumn{3}{|c|}{$\begin{array}{l}\text { Note: } \mathrm{ASA}=\text { acetylsalicylic acid, } \mathrm{CrCl}=\text { creatinine clearance, } \mathrm{CYP}=\text { cytochrome } \mathrm{P} 450, \mathrm{NSAID}=\text { nonsteroidal anti-inflammatory drug, } \mathrm{P} \text {-gp }=\mathrm{P}-\mathrm{glycoprotein} . \\
\text { *Sources: Mueck and associates, } \\
\text { clinical-table). } \\
\text { tBased on expert opinion; this guidance must be interpreted in patient-specific context. } \\
\text { fThe list of potential moderate CYP3A4 inhibitors is not exhaustive; for a more comprehensive list, see the list prepared by the Department of Medicine, Indiana } \\
\text { University (http://medicine.iupui.edu/clinpharm/ddis/clinical-table). }\end{array}$} \\
\hline
\end{tabular}




\section{Management of bleeding}

Unlike warfarin, rivaroxaban has no specific reversal agent. Although phase II studies have shown that andexanet alfa may be an effective option for reversing factor Xa inhibitors (e.g., rivaroxaban, apixaban), this possibility has yet to be confirmed in phase III clinical trials. ${ }^{10}$ Considering rivaroxaban's short half-life, minor bleeding can be managed symptomatically (e.g., local compression, nasal packing) or by temporary discontinuation of the drug. For patients with major bleeding, some experts recommend the use of antifibrinolytic agents (e.g., tranexamic acid, $\varepsilon$-aminocaproic acid). ${ }^{11}$ For life-threatening bleeding, nonspecific hemostatic agents such as fourfactor prothrombin complex concentrate are recommended, on the basis of expert opinion and small randomized controlled trials. ${ }^{11}$ However, treatment with prothrombin complex concentrates is associated with a marked prothrombotic risk and should be reserved for truly life-threatening bleeding. Dialysis is generally not recommended, even for life-threatening bleeding, because 95\% of rivaroxaban is bound to protein. ${ }^{1}$

\section{References}

1. Samama MM, Contant G, Sprio T, et al. Laboratory assessment of rivaroxaban: a review. Thromb J 2013;11:11.

2. Samama MM, Contant G, Spiro TE, et al. Evaluation of the antifactor Xa chromogenic assay for the measurement of rivaroxaban plasma concentrations using calibrators and controls. Thromb Haemost 2012;107:379-87.

3. Wasserlauf G, Grandi SM, Filion KB, et al. Meta-analysis of rivaroxaban and bleeding risk. Am J Cardiol 2013;112:454-60.

4. Ruíz-Giménez N, Suárez C, González R, et al. Predictive variables for major bleeding events in patients presenting with documented acute venous thromboembolism. Findings from the RIETE Registry. Thromb Haemost 2008;100:26-31.

5. Wakasugi H, Yano I, Ito T, et al. Effect of clarithromycin on renal excretion of digoxin: interaction with P-glycoprotein. Clin Pharmacol Ther 1998;64:123-8.
6. Gnoth MJ, Buetehorn U, Muenster U, et al. In vitro and in vivo P-glycoprotein transport characteristics of rivaroxaban. J Pharmacol Exp Ther 2011;338:372-80.

7. Mueck W, Kubitza D, Becka M. Co-administration of rivaroxaban with drugs that share its elimination pathways: pharmacokinetic effects in healthy subjects. $\mathrm{Br} J$ Clin Pharmacol 2013;76:455-66.

8. Holbrook AM, Pereira JA, Labiris R, et al. Systematic overview of warfarin and its drug and food interactions. Arch Intern Med 2005; 165:1095-106

9. Xarelto [product monograph]. Mississauga (ON): Bayer Inc.; 2015. Available: http://omr.bayer.ca/omr/online/xarelto-pm-en. pdf (accessed 2016 Jan. 16).

10. Siegal DM, Curnutte JT, Connolly SJ, et al. Andexanet alfa for the reversal of factor Xa inhibitor activity. $N$ Engl J Med 2015;373:2413-24.

11. Koscielny J, Rutkauskaite E. Rivaroxaban and hemostasis in emergency care. Emerg Med Int 2014;2014:935474.

Affiliations: Department of Medicine (Fralick, Juurlink, Marras), University of Toronto, Toronto, Ont.; Institute for Clinical Evaluative Sciences (Juurlink), Toronto, Ont.; Division of Internal Medicine (Juurlink), Sunnybrook Health Sciences Centre, Toronto, Ont.; Division of Respirology (Marras), Department of Medicine, University Health Network and Mount Sinai Hospital, Toronto, Ont.

Contributors: All of the authors participated in interpreting and analyzing the data collected for the case report and in writing and revising the article, gave final approval of the version to be published and agreed to act as guarantors of the work.

The section Cases presents brief case reports that convey clear, practical lessons. Preference is given to common presentations of important rare conditions, and important unusual presentations of common problems. Articles start with a case presentation (500 words maximum), and a discussion of the underlying condition follows (1000 words maximum). Visual elements (e.g., tables of the differential diagnosis, clinical features or diagnostic approach) are encouraged. Consent from patients for publication of their story is a necessity. See information for authors at www.cmaj.ca. 